KURZ GEMELDET

Allergy News

\section{Giftiges Feuer}

orscher der Technischen Universität

Wien untersuchten den Schnee vor und nach der Neujahrsnacht 2007/2008 in Saalbach, Tirol. Dort hatte es während des Jahreswechsels geschneit. Die Flocken hatten die Luft ausgewaschen, sodass Stof$\mathrm{fe}$, die mit dem Silvesterfeuerwerk in die Atmosphäre gelangt waren, auf der Erde nachweisbar waren. Wasserlösliche Salze des giftigen Erdalkalimetalls Barium wurden in über 600-fach erhöhter Konzentration gefunden. Auch ein Anstieg der Strontium- und der Arsenkonzentrationen wurde gemessen. Cäsium und Kobalt wurden in geringen Spuren nachgewiesen. Es ist denkbar, dass die Bariumaerosole bei Zuschauern in unmittelbarer Nähe des

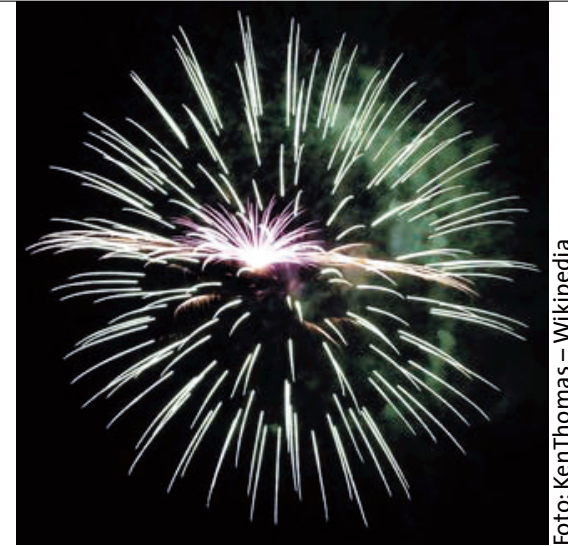

Feuerwerks Atembeschwerden auslösen. So wurde in einer anderen Studie nachgewiesen, dass am 4. Juli, dem ebenfalls von Feuerwerk begleiteten Nationalfeiertag der USA, die Zahl der Asthmaanfälle signifikant erhöht ist.

Steinhauser G et al. Atmos Environ 2008; 42: 8616-22

\section{Leben dank guter Luft}

_ in Rückgang der Luftverschmutzung zahlt sich für die Bevölkerung aus durch gewonnene Lebensjahre. Eine Studie ermittelte, dass die Lebenserwartung in 51 US-Städten von 1980 bis 2000 um fast drei Jahre angestiegen ist. Ein signifikanter Anteil von fünf gewonnenen Lebensmonaten geht nach den Berechnungen der Forscher allein auf die Tatsache zurück, dass die Luftqualität in den untersuchten Städten in den letzten Jahren kontinuierlich verbessert werden konnte. Es wird vermutet, dass die sauberere Luft insbesondere zu einem Rückgang kardiovaskulärer und kardiopulmonaler Erkrankungen geführt hat.

Krewski D. N Engl J Med 2009; 360: 413-5

D ie Stickstoffmonoxidkonzentration in der Atemluft von Asthmatikern korreliert mit dem Grad der Atemwegsentzündung. Zunehmend häufiger werden deshalb NO-Messungen ergänzend zu den klassischen Kontrollparametern Symptomatik und Lungenfunktion eingesetzt. In einer großen, auf ein Jahr angelegten randomisierten Studie an zehn innerstädtisch gelegenen US-amerikanischen Asthmazentren wurde nun überprüft, ob das zusätzliche NO-Monitoring die Effektivität der Behandlung im Vergleich zu einer entsprechend den nationalen Richtlinien

durchgeführten Asthmatherapie verbessert. Einbezogen waren 546 überwiegend atopische Asthmakranke im Alter zwischen 12 und 20 Jahren. Bei den meisten Patienten konnte mit dem Standard-Behandlungsalgorithmus eine gute Krankheitskontrolle erreicht werden. Bei zusätzlicher NO-Messung erhielten die Patienten im Durchschnitt mehr Glukokortikoide und lang wirksame $\beta$-Agonisten, allerdings ohne verbesserte Werte bei Symptomatik und Lungenfunktion. $b k$

Szefler SJ et al. Lancet 2008; 372: 1065-72

\title{
Mäuse-induziertes Wheezing
}

\begin{abstract}
$\mathrm{n}$ den städtischen Regionen der USA
steigt die Asthmainzidenz stark an. Als

Allergenquellen spielen neben Hausstaubmilben und Kakerlaken auch Mäuse ein Rolle. In einer prospektiven Studie mit knapp 500 Risikokindern in Boston sollte der Zusammenhang quantifiziert werden. Danach erhöhte eine von den Eltern berichtete Mäuseexposition im ersten Lebensjahr das Wheezing-Risiko im ersten Lebensjahr, bei weiterer Exposition blieb es erhöht. Keinen Einfluss hatte der Mausallergenkontakt in früher Kindheit auf die Prävalenz

Foto: George Shuklin
\end{abstract}

von Asthma, Ekzem oder allergischer Rhinitis im Alter von sieben Jahren. Nachweisbare Mausallergenkonzentrationen im Säuglingsalter waren aber mit einer Verdoppelung der Zahl atopisch Sensibilisierter (Reaktion auf mehr als ein Allergen) im Schulalter assoziiert. Ob diese Zusammenhänge nur bei genetisch belasteten Risikokindern zu finden sind oder sich auch auf die Normalbevölkerung übertragen lassen, ist noch unklar.

$b k$

西

\section{(1)}

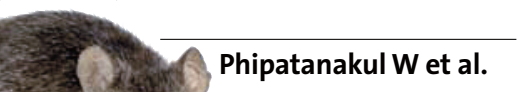
Allergy 2008; 63: 1512-8

\section{Allergie gegen Rhodium}

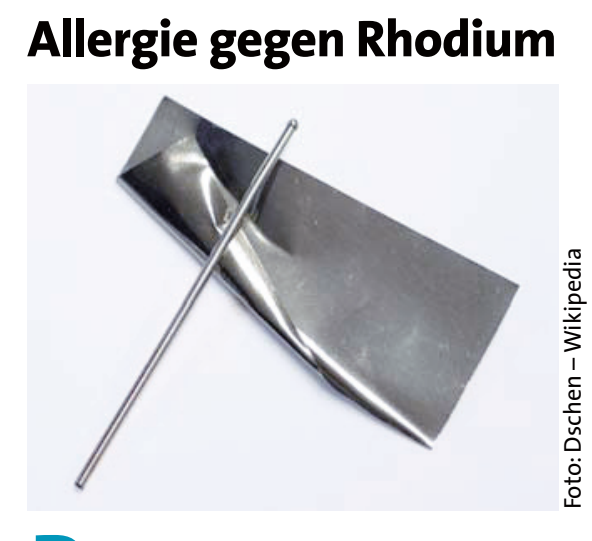

hodium wird vor allem als Katalysator- oder Beschichtungsmaterial eingesetzt. Das Element zählt zur Gruppe der Platinmetalle, von denen bereits einige als Auslöser für allergische Reaktionen bekannt sind. Rhodium gehörte bislang nicht dazu. Das Bochumer BGFA hat jetzt den ersten Fall einer Soforttypallergie gegen Rhodiumsalze bei einem 27-jährigen Galvaniseur beschrieben. Der Patient reagierte im Pricktest und bei der bronchialen Provokation auf das Rhodiumsalz $\mathrm{Na}_{3} \mathrm{RhCl}_{6}$. Spezifische IgE-Antikörper gegen das Rhodiumsalz konnten nicht nachgewiesen werden. Dies spricht aber nicht gegen einen IgE-vermittelten Mechanismus, denn auch bei Platinsalzen gelingt der In-vitro-IgE-Nachweis nur mit geringer Sensitivität.

Merget et al. BGFA-Info 2008 (3): 6-9 